\title{
KINEMATICS OF TABLE TENNIS TOPSPIN FOREHANDS: EFFECTS OF PERFORMANCE LEVEL AND BALL SPIN
}

\author{
Yoichi lino and Takeji Kojima
Department of Life Sciences, Graduate School of Arts and Sciences, the University of Tokyo, Tokyo, Japan

KEY WORDS: table tennis, forehand, contribution, acceleration

INTRODUCTION: The topspin forehand in table tennis is an effective offensive shot especially against backspin balls. It would be interesting for coaches and players to compare the kinematics of the stroke between different performance level players and between different ball spins. The purpose of this study was to clarify whether performance level and ball spin affect the kinematics of the table tennis topspin forehand.

METHOD: Nine advanced and 8 intermediate male collegiate table tennis players hit topspin forehands against light and heavy backspins. Five high speed video cameras were used to record their strokes at 200 frames per second. Contributions of joint rotations to the racket speed (Sprigings et al.,1994), the racket kinematics at ball impact, the time when the racket acquired the $25 \%$ value of the racket speed at impact relative to impact $\left(t_{0.25}\right)$ and the maximum slope of the racket speed-time curve $\left(s_{\max }\right)$ were determined. A two-way repeated measure ANOVA tested the significance of the effects $(P<0.01)$.

RESULTS: The advanced players showed a significantly larger contribution of lower trunk axial rotation to the racket speed at impact $(P=0.009)$ and had a significantly larger value of $s_{\max }(\mathrm{P}=0.004)$ and tended to have a later time of $t_{0.25}(\mathrm{P}=0.034)$ than the intermediate players (Table 1). The racket speed at impact was not significantly different between the two subject groups $(P=0.111)$. The players adjusted the racket face angle rather than the inclination of the racket path at impact to the different ball spins.

Table 1 Racket speed at impact and the variables related to racket acceleration

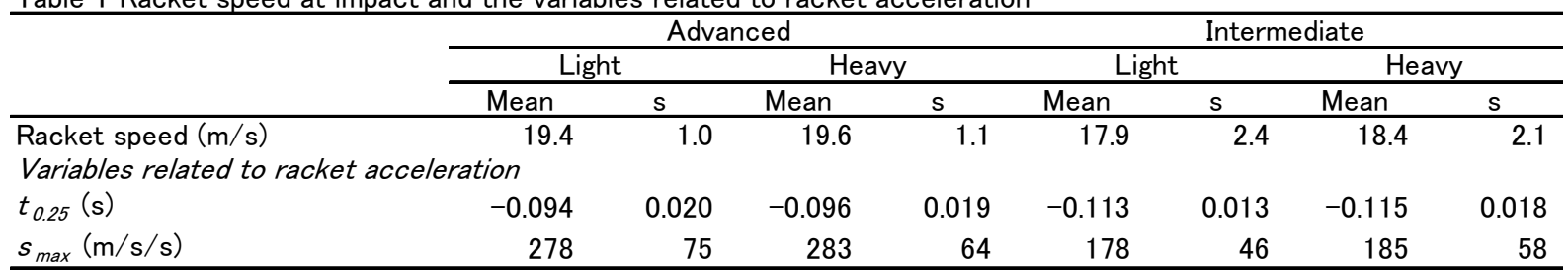

DISCUSSION: This study highlighted the importance of the variables related to racket acceleration rather than the racket speed at impact for the performance of the forehand against backspin balls. A sufficient use of lower trunk axial rotation may help a player accelerate the racket in a less time.

CONCLUSION: This study clarified the effects of performance level and ball spin on the kinematics of the table tennis forehand. The results suggest that the ability to accelerate the racket in a less time is one of the important factors for the forehand against backspins.

\section{REFERENCES:}

Sprigings E., Marshall R., Elliott B. \& Jennings L. (1994). A three-dimensional kinematic method for determining the effectiveness of arm segment rotations in producing racquet-head speed. Journal of Biomechanics, 27, 245-254.

Acknowledgement This study was funded by the Japan Table Tennis Association. The authors thank Dr. T. Ohtsuki and Dr. K. Kudo for their support in the experiment. 